\title{
Design of an Inertially Counterbalanced $Z$-Nanopositioner for High-Speed Atomic Force Microscopy
}

\author{
Y. K. Yong, Member, IEEE, and S. O. R. Mohemani, Fellow, IEEE
}

\begin{abstract}
In many conventional atomic force microscopes (AFMs), one of the key hurdles to high-speed scanning in constantforce contact mode is the low-feedback control bandwidth of the $Z$-axis loop. This paper presents the design of a fast $Z$-nanopositioner to overcome this limitation. The $Z$-nanopositioner has its first resonant mode at $60 \mathrm{kHz}$ and a travel range of $5 \mu \mathrm{m}$. It consists of a piezoelectric stack actuator and a diaphragm flexure. The flexure serves as a linear spring to preload the actuator and to prevent it from getting damaged during high-speed operations. The $Z$-nanopositioner is mounted to an $X Y$-nanopositioner. To avoid exciting the resonance of the $X Y$-nanopositioner, an inertial counterbalance configuration was incorporated in the design of the $Z$-nanopositioner. With this configuration, the resonances of the $X Y$-nanopositioner were not triggered. A closed-loop vertical control bandwidth of $6.5 \mathrm{kHz}$ is achieved. High-speed constant-force contact-mode images were recorded at a resolution of $200 \times 200$ pixels at 10, 100, and $200 \mathrm{~Hz}$ line rates without noticeable image artifacts due to insufficient control bandwidth and vibrations. Images were also recorded at 312 - and $400-\mathrm{Hz}$ line rates. These images do not show significant artifacts. These line rates are much higher than the closed-loop bandwidth of a conventional AFM in which this nanopositioner was tested.
\end{abstract}

Index Terms-Atomic force microscope, flexure, nanopositioner, vertical bandwidth.

\section{INTRODUCTION}

$\mathbf{T}$ HE invention of scanning tunneling microscope (STM) [1] and atomic force microscope (AFM) [2] in the 1980s has revolutionized research in various areas of nanotechnology, including material sciences [3], [4], nanolithography and nanopatterning [5]-[7], nanometrology [8], bionanotechnology [9], [10], semiconductor manufacturing [5], [6], and high-density data storage systems [11], [12]. Unlike STMs, which require the measured surface to be electrically conductive, AFMs have the capability to investigate both conductive and insulator surfaces. AFMs can be operated either in static or dynamic mode. Details of the dynamic (or noncontact) mode will not be discussed in this paper but extensive reviews can be found in [4], [13],

Manuscript received November 1, 2011; accepted December 7, 2012. Date of publication December 12, 2012; date of current version March 6, 2013. This work was supported in part by the Australian Research Council and in part by the University of Newcastle, Australia. The review of this paper was arranged by Associate Editor P. J. Burke.

The authors are with the School of Electrical Engineering and Computer Science, the University of Newcastle, N.S.W. 2308, Australia (e-mail: Yuenkuan.Yong@newcastle.edu.au; reza.moheimani@newcastle.edu.au).

Color versions of one or more of the figures in this paper are available online at http://ieeexplore.ieee.org.

Digital Object Identifier 10.1109/TNANO.2012.2233749
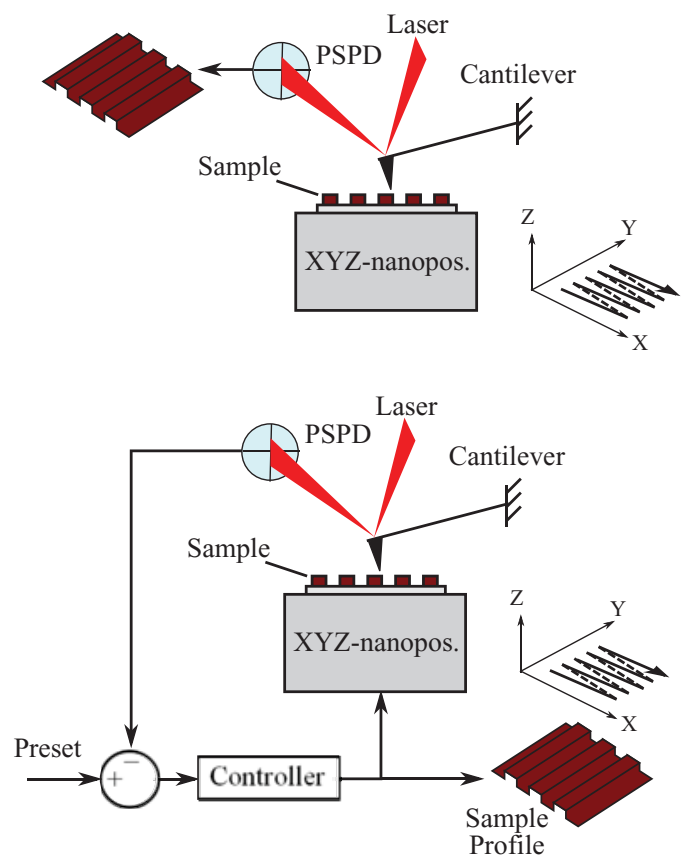

Fig. 1. (Top) Constant-height contact-mode AFM. (Bottom) Constant-force contact-mode AFM.

and [14]. In the static mode (or contact mode), a cantilever with a sharp tip at one end is slowly brought in contact with a sample surface. The tip-sample interaction force, typically in the order of subnano-Newtons, causes the cantilever to deflect. Surface topography is obtained by raster scanning the sample under the tip while simultaneously measuring the cantilever's deflection using a laser and a position sensitive photodiode (PSPD). In the constant-height contact mode, the topographic information is obtained by recording the cantilever's deflection directly. In the constant-force contact mode, the deflection is used as a control parameter for a vertical feedback controller which maintains the interaction force at a constant value [4]. In this mode, the surface topography is constructed from the control signal (see Fig. 1). The main disadvantage of the constant-force contact mode is its low scan speed. The two key factors that limit the scan speed are the low resonance frequency of an $X Y Z$-nanopositioner that is used to position the sample, and the insufficient closed-loop bandwidth of the $Z$-axis feedback controller [15]-[18]. For example, a conventional AFM piezoelectric tube scanner has a resonance frequency of several hundred $\mathrm{Hz}$, which limits the vertical feedback loop bandwidth, typically 


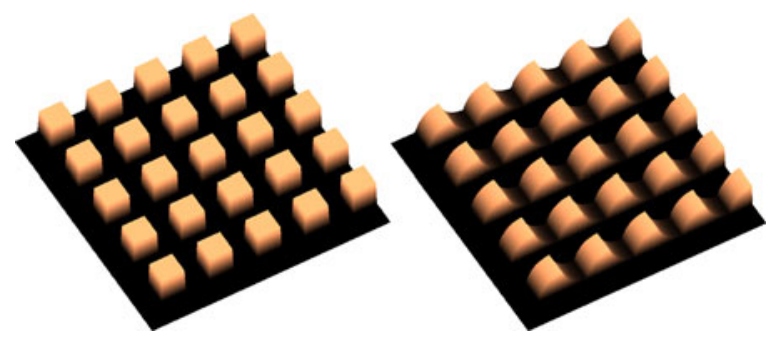

Fig. 2. Simulated image artifact associated with an insufficient vertical feedback bandwidth. (Left) Perfect image of a testing grating with sharp features. (Right) Image with artifact.

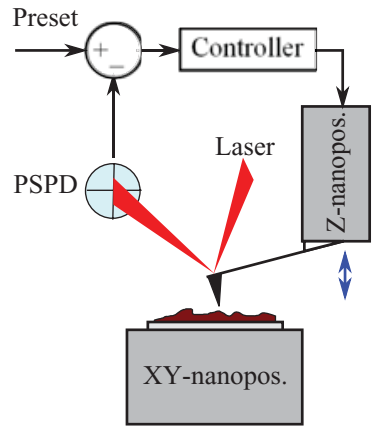

Sample-scan

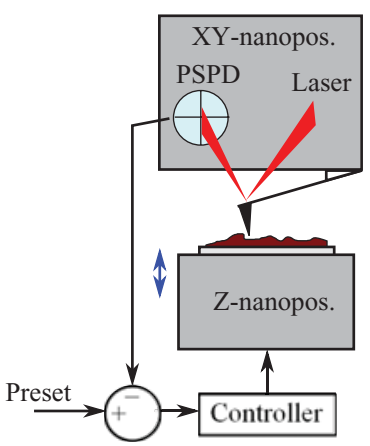

Probe-scan
Fig. 3. Sample-scan and probe-scan configurations.

to the order of $100 \mathrm{~Hz}$ [17]. When imaging a sample with sharp features at high speed (beyond 5-Hz line rates), the low vertical closed-loop bandwidth will cause similar image artifacts as shown in Fig. 2 where a "smudging" effect on the sharp edges of the sample features will be observed [17], [19]. To avoid this image artifact, some researchers conduct high-speed scanning in the constant-height contact mode where the $Z$ feedback loop is deactivated [20], [21]. This scan mode may result in excessive forces between the tip and sample, which tends to damage the cantilever, sample, or both. The constant-height contact mode is often not suitable for imaging soft samples such as biological cells.

$X Y$-nanopositioners are heavier structures; therefore, they have lower resonance frequencies than that of $Z$-nanopositioners [20], [22]. During high-scan speed operations, the dynamics of a $Z$-nanopositioner tends to trigger the resonances of an $X Y$-stage. To decouple the dynamics, the $X Y$ - and $Z$-nanopositioners are mechanically separated [17], [23], [24]. This will allow one to devise a very high-bandwidth $Z$-nanopositioner without the influence of the $X Y$-resonant modes. With the $X Y$ and $Z$-nanopositioners separated mechanically, a sample can be imaged in either sample-scan or probe-scan mode (see Fig. 3). When operated in the sample-scan mode, an $X Y$-nanopositioner is used to move the sample over an area in a raster pattern, and a $Z$-nanopositioner is used to position the cantilever and to adjust the interaction force between the tip and sample. This configuration may require complicated alignment of the optics and laser in order to accurately measure the deflection of the cantilever [24]. In the probe-scan mode, it is the cantilever that

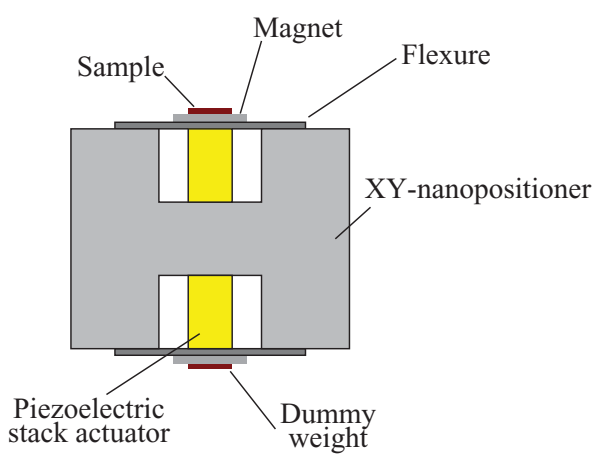

Fig. 4. Dual-mounted $Z$-nanopositioner for counterbalancing impulsive forces.

is moved in a raster pattern using an $X Y$-nanopositioner. The $Z$-nanopositioner is positioned under the cantilever and is used to adjust the distance of the sample from the cantilever. Although a high-bandwidth $Z$-nanopositioner can be designed without being limited by the $X Y$ resonances, the $X Y$-nanopositioner is forced to carry the weight of a scanning unit (which involves relatively heavy mechanical components that are used to assemble the laser, PSPD, and cantilever). This will lead to a reduction of the mechanical bandwidth of the $X Y$-nanopositioner which in turn limits the scan speed of the AFM.

In this paper, we report a design that involves a $Z$-nanopositioner which is mounted on an $X Y$-nanopositioner, and the system is operated such that images are obtained in the samplescan mode as shown in Fig. 1. The sample is moved in a raster trajectory using the assembled $X Y Z$-nanopositioner. The proposed $Z$-nanopositioner is used to regulate the tip-sample interaction force. With this arrangement, complications that may arise due to the misalignment of the laser, PSPD, and cantilever are avoided. However, it is a challenging task to simultaneously obtain the high mechanical bandwidth for both $X Y$ - and $Z$-nanopositioners. This is because the impulsive forces generated from the rapid movement of the $Z$-axis piezoelectric stack actuator may excite the resonant modes of the $X Y$ nanopositioner [13], [23]. To counterbalance the impulsive force, a dual-mounted $Z$-nanopositioner configuration [13], [17], [20] is used in this study. A pair of piezoelectric stack actuators are displaced concurrently with the same magnitude but in opposite directions. Furthermore, in order to balance the sample weight, a dummy mass is placed at the opposite end of the $Z$-nanopositioner (see Fig. 4).

Flexures have been commonly used in many nanopositioners to generate repeatable and smooth motions on the nanometer scale [25]-[29]. Diaphragm-like flexures have been used to provide accurate out-of-plane motion for applications such as flexible couplings [30] and positioning of an objective lens in microscopes [31]. In this study, a diaphragm flexure is designed to guide the motions of the $Z$-axis piezoelectric stack actuator. It is also used as a linear spring to provide the necessary preload to the actuator.

The remainder of this paper proceeds as follows. Section II discusses the design of a single-mounted $Z$-nanopositioner. Analytical stiffness derivation of a diaphragm flexure which is 
(a)

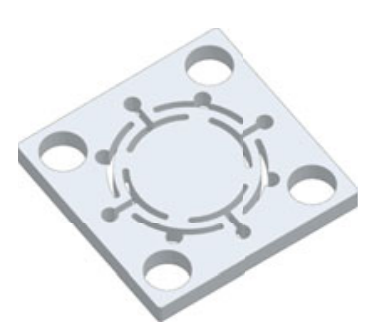

(c)

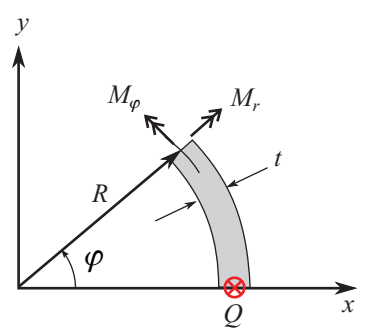

(b)

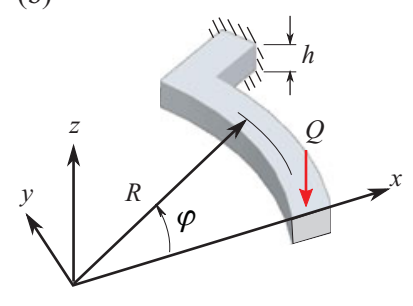

(d)

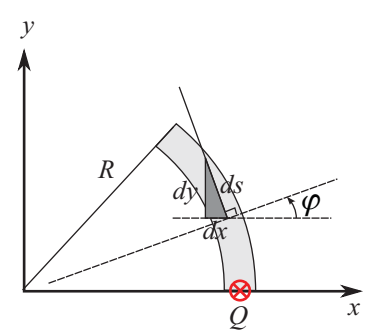

Fig. 5. Diaphragm flexure of the $Z$-nanopositioner. (a) Diaphragm flexure. (b) Curved-beam model. (c) Cross-sectional bending and torsional moments of the beam. (d) Beam element.

used to construct the $Z$-nanopositioner is presented. The resonance frequency of the $Z$-nanopositioner is estimated analytically as well as using ANSYS (a finite-element-analysis package). Analytical estimation of the travel range of the nanopositioner is also presented. Section III presents a dual-mounted $Z$ nanopositioner which is used to realize the inertial counterbalance technique. Experimental setup and results of the proposed dual-mounted $Z$-nanopositioner are presented in Section IV. Section V reports the imaging performance of the dual system. Finally, Section VI concludes the paper.

\section{Single-Mounted $Z$-NANOPOSITIONER}

Here, a single-mounted $Z$-nanopositioner is first studied in order to obtain an in-depth understanding of the static and dynamic behavior of the design. The following shows the procedure of obtaining analytical stiffness and resonance frequency equations of the design.

A diaphragm-like structure which consists of eight fixed free curved beams, illustrated in Fig. 5, is used to construct the $Z$-nanopositioner. The combination of flexures provides the necessary preload to the piezoelectric stack actuator and eliminates the parasitic rotational motions of the $Z$-nanopositioner. The main advantages of diaphragm-like flexures are their compactness and simplicity [32]. The curved beams can be accurately manufactured using the wire electric discharge machine (WEDM).

\section{A. Analytical Stiffness Equations}

The out-of-plane stiffness of a flexure can be derived by using the strain energy method [33] and Castigliano's second theorem [20], [28], [34]. As shown in Fig. 5(b), the curved beam is clamped at one end and a load $Q$ is applied at the other. The total strain energy of the curved beam flexure (neglecting the

influence of shear force) is

$$
U=\int_{0}^{L} \frac{M_{r}^{2}}{2 E I} d s+\int_{0}^{L} \frac{M_{\phi}^{2}}{2 G J} d s
$$

where $M_{r}$ and $M_{\phi}$ as shown in Fig. 5(c) are the cross-sectional bending and torsional moments, which vary with $\phi, E$ is Young's modulus, $G=E / 2(1+v)$ is the shear modulus, $v$ is Poisson's ratio, $I=h^{3} t / 12$ is the second moment of inertia, $J=\left(h t^{3}+\right.$ $\left.h^{3} t\right) / 12$ is the polar moment of inertia, $d s$ is the length of a beam element, and $L$ is the length of the beam. By using Castigliano's second theorem, the deflection $\delta_{z}$ of the curved beam flexure can be calculated by taking the partial derivative of $U$, that is

$$
\delta_{z}=\frac{\partial U}{\partial Q}=\frac{1}{E I} \int_{0}^{L} M_{r} \frac{\partial M_{r}}{\partial Q} d s+\frac{1}{G J} \int_{0}^{L} M_{\psi} \frac{\partial M_{\psi}}{\partial Q} d s .
$$

The equilibrium equations for moment along the $x$ and $y$ directions are [35]

$$
\begin{aligned}
M_{r} \cos \phi-M_{\phi} \sin \phi+Q y & =0 \\
M_{r} \sin \phi+M_{\phi} \cos \phi+Q(R-x) & =0 .
\end{aligned}
$$

By solving for $M_{r}$ and $M_{\phi}$, we have

$$
\begin{aligned}
& M_{r}=-Q y \cos \phi-Q(R-x) \sin \phi \\
& M_{\phi}=Q y \sin \phi-Q(R-x) \cos \phi .
\end{aligned}
$$

To perform the integration of (2) over the variable $x$ in the Cartesian coordinates, the following procedure is employed [35]. The equation of the curved beam is defined as

$$
\begin{aligned}
x^{2}+y^{2} & =R^{2} \\
y & =\sqrt{R^{2}-x^{2}}
\end{aligned}
$$

where $R$ is the radius of curvature of the beam. By differentiating (8), we have

$$
\frac{d y}{d x}=-\frac{x}{\sqrt{R^{2}-x^{2}}} .
$$

As shown in Fig. 5(c), the length of a beam element $d s$ is

$$
d s=\sqrt{d x^{2}+d y^{2}}=d x \sqrt{1+\left(\frac{d y}{d x}\right)^{2}} .
$$

And $\sin (\phi)$ and $\cos (\phi)$ are expressed as

$$
\begin{aligned}
& \sin \phi=\frac{d x}{d s}=\frac{1}{\sqrt{1+\left(\frac{d y}{d x}\right)^{2}}} \\
& \cos \phi=\frac{d y}{d s}=\frac{-d y / d x}{\sqrt{1+\left(\frac{d y}{d x}\right)^{2}}} .
\end{aligned}
$$

By substituting (5), (6), (10)-(12) and $\partial M_{r} / \partial Q, \partial M_{\phi} / \partial Q$ into (2), and by performing integration from $x=R \cos (\pi / 4)$ (where $\pi / 4$ is the angle of the beam) to $x=R$, we have

$$
\delta_{z}=Q R^{2}\left(\frac{T_{1}}{E I}+\frac{T_{2}}{G J}\right)
$$


TABLE I

Dimensions AND MATERIAL PROPERTIES OF THE FLEXURE AND PIEZOELECTRIC STACK ACTUATOR

\begin{tabular}{lr}
\hline \hline Diaphragm Flexure & Values \\
\hline Radius of curvature, $R(\mathrm{~mm})$ & 2.99 \\
Thickness, $t(\mathrm{~mm})$ & 0.38 \\
Height, $h(\mathrm{~mm})$ & 1.1 \\
Effective mass, $m_{f}(\mathrm{~g})$ & 0.32 \\
Stiffness, $k_{f}(\mathrm{~N} / \mu \mathrm{m})$ & $5.17^{\mathrm{a}}$ \\
Young's Modulus, $E(\mathrm{GPa})$ & 72 \\
Poisson's ratio, $v$ & 0.33 \\
Density, $\rho\left(\mathrm{kg} / \mathrm{m}^{3}\right)$ & 2770 \\
\hline Piezo & Values \\
\hline Dimension $(\mathrm{mm})$ & $3 \times 3 \times 6$ \\
Mass, $m_{p}(\mathrm{~g})$ & 0.41 \\
Stiffness, $k_{p}(\mathrm{~N} / \mu \mathrm{m})$ & 65 \\
Young's Modulus, $E_{p}(\mathrm{GPa})$ & 45 \\
Poisson's ratio, $v_{p}$ & 0.3 \\
Capacitance, $C_{p}(\mathrm{nF})$ & 70 \\
Travel range @ $200 \mathrm{~V}(\mu \mathrm{m})$ & 5.5 \\
\hline Support structure $(\mathrm{XY}$-stage $)$ & Values \\
\hline Mass, $m_{s}(\mathrm{~g})$ & 5.72 \\
Stiffness, $k_{s}(\mathrm{~N} / \mu \mathrm{m})$ & $81^{\mathrm{b}}$ \\
Density, $\rho\left(\mathrm{kg} / \mathrm{m}^{3}\right)$ & 7500 \\
\hline \hline a Obtained using $(16)$. & \\
${ }^{\mathrm{b}}$ Obtained using FEA.
\end{tabular}

where $T_{1}$ and $T_{2}$ are

$$
\begin{aligned}
& T_{1}=-\int_{\frac{R}{\sqrt{2}}}^{R} \frac{1}{\sqrt{\frac{R^{2}}{R^{2}-x^{2}}}} d x \\
& T_{2}=-\int_{\frac{R}{\sqrt{2}}}^{R} \frac{R-x}{(R+x) \sqrt{\frac{R^{2}}{R^{2}-x^{2}}}} d x .
\end{aligned}
$$

From (13), the stiffness of the curved beam is $K_{z}=Q / \delta_{z}$. The diaphragm flexure consists of eight curved beams arranged in parallel. Therefore, the total stiffness of the flexure is

$$
K_{f}=\frac{8 Q}{\delta_{z}} .
$$

For the chosen flexure dimensions and material as documented in Table I, $K_{f}$ is calculated to be $5.17 \mathrm{~N} / \mu \mathrm{m}$.

\section{B. Resonance Frequency}

The resonance frequency of the single-mounted $Z$-nanopositioner can be estimated by modeling it as a two-degree-offreedom (DOF) spring-mass-damper system [36] as shown in Fig. 6. In this model, the upper half of the piezoelectric stack actuator is lumped to the effective mass of the flexure and sample, and the lower half of the actuator is lumped to the effective mass of the support structure, that is the $X Y$ nanopositioner. Also, $c_{1}$ and $c_{2}$ represent the effective damping coefficients, and $k_{1}$ and $k_{2}$ are the effective stiffnesses. We have $m_{1}=m_{p} / 2+m_{s}, m_{2}=m_{p} / 2+m_{f}, c_{1}=c_{s}, c_{2}=c_{p}+c_{f}$, $k_{1}=k_{s}$, and $k_{2}=k_{p}+k_{f}$. The subscripts $p, f$, and $s$ stand for piezoelectric stack actuator, diaphragm flexure, and support structure, respectively. By using the lumped spring-massdamper system, two resonances $\left(f_{1}\right.$ and $\left.f_{3}\right)$ and one antireso-

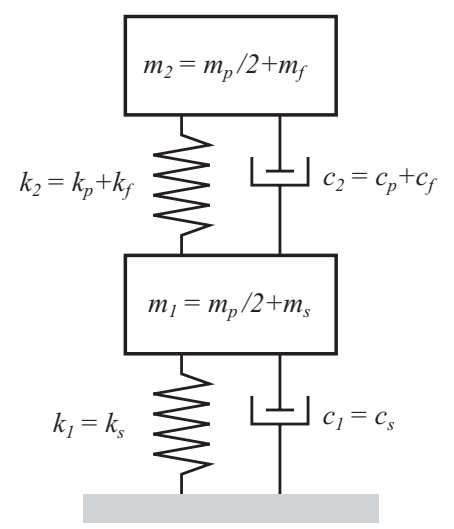

Fig. 6. 2-DOF spring-mass model of the $Z$-nanopositioner where $m, c$, and $k$ are mass, damping coefficient, and stiffness, respectively. The subscripts $p$, $f$, and $s$ are referred to the piezoelectric stack actuator, diaphragm flexure, and support structure (the $X Y$-nanopositioner), respectively.

nance $\left(f_{2}\right)$ can be obtained [36], as

$$
\begin{aligned}
& f_{1} \approx \frac{1}{2 \pi} \sqrt{\frac{k_{1}}{m_{1}+m_{2}}} \\
& f_{2} \approx \frac{1}{2 \pi} \sqrt{\frac{k_{1}}{m_{1}}} \\
& f_{3} \approx \frac{1}{2 \pi} \sqrt{\frac{\left(m_{1}+m_{2}\right) k_{2}}{m_{1} m_{2}}} .
\end{aligned}
$$

Here, $f_{1}$ is the vertical resonant mode of the $X Y$-nanopositioner, and $f_{3}$ is the resonant mode of the preloaded piezoelectric stack actuator with flexures. The calculated resonance frequencies $f_{1}$ and $f_{3}$ of the single-mounted $Z$-nanopositioner design are 17.8 and $61 \mathrm{kHz}$, respectively.

\section{Finite-Element Analysis}

ANSYS was used to perform finite-element (FE) analysis of the $Z$-nanopositioner. The flexure is deformed using a piezoelectric stack actuator that generates the requisite vertical displacement. A magnet is glued onto the flexure as illustrated in Fig. 7. The magnet's function is to hold a metal sample holder. It has a diameter of $5 \mathrm{~mm}$ and a thickness of $1 \mathrm{~mm}$. The flexure is made of aluminum alloy (Al7075). The piezoelectric actuator is a Noliac SCMAP06 stack actuator. Dimensions and material properties of the actuator, which were used to construct the ANSYS model, can be found in Table I.

ANSYS simulations predicted that the first vertical resonance frequency of the $X Y$-nanopositioner is at $19.7 \mathrm{kHz}$. The vertical resonant mode of the $Z$-nanopositioner appears at $65 \mathrm{kHz}$ (see Fig. 7). The simulated stiffness along the $Z$-axis is $5.15 \mathrm{~N} / \mu \mathrm{m}$. The analytical resonance frequencies and stiffness are in reasonable agreement with the FE simulations.

\section{Travel Range}

To estimate the travel range of the nanopositioner, we may use the formulation $\Delta L=\Delta L_{o} \cdot k_{p} /\left(k_{f}+k_{p}\right)$ [37], [38], where $\Delta L$ is the displacement with external spring load, $\Delta L_{o}$ is the 


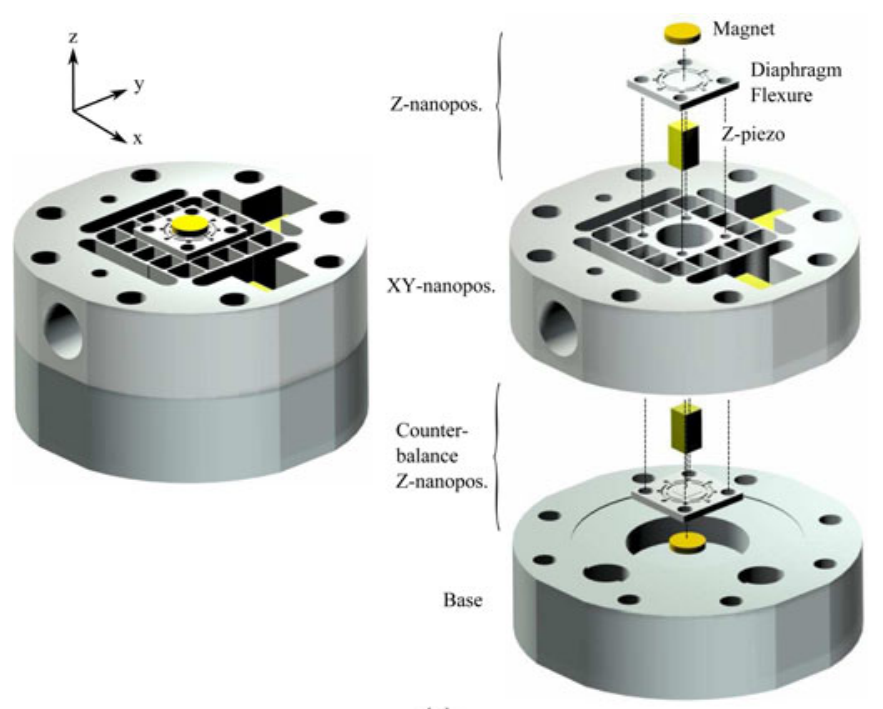

(a)

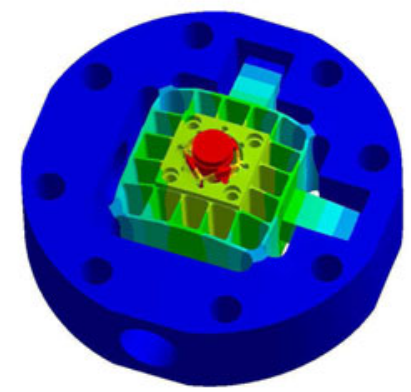

(b)

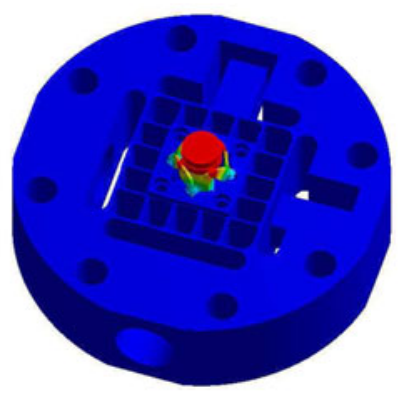

(c)
Fig. 7. $Z$-nanopositioner design. (a) Exploded view of the $Z$-nanopositioner. (b) Finite-element simulated first vertical resonant mode of the $X Y$ nanopositioner at $19.7 \mathrm{kHz}$. (c) Finite-element simulated first vertical resonant mode of the $Z$-nanopositioner at $65 \mathrm{kHz}$.

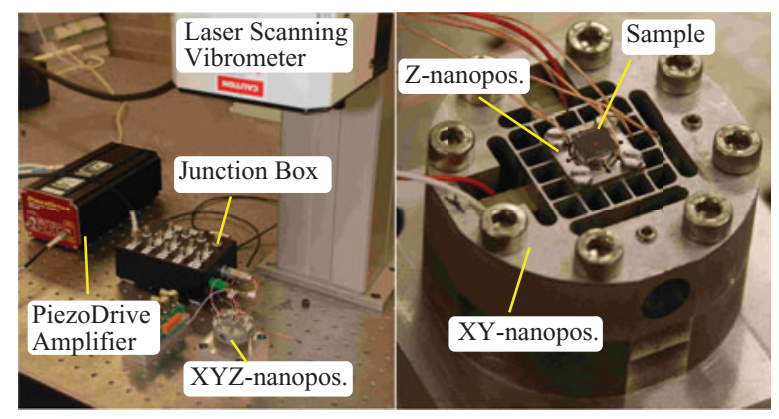

Fig. 8. Experimental setup of the nanopositioner. The $Z$-nanopositioner is mounted to the $X Y$-nanopositioner.

maximum nominal displacement of the piezoelectric stack actuator without an external spring load, $k_{f}$ is the flexure stiffness and $k_{p}$ is the piezoelectric stack actuator stiffness. The estimated displacement of the $Z$-nanopositioner is $5.1 \mu \mathrm{m}$.

\section{DUAL-MOUnTED Z-NANOPOSITIONER}

The $Z$-nanopositioner is mounted on top of a parallelkinematics $X Y$-nanopositioner. The $X Y$ travel range of the nanopositioner, illustrated in Fig. 8, is $6.5 \mu \mathrm{m} \times 6.5 \mu \mathrm{m}$. Details of the $X Y$-stage design and its properties are discussed in (a)
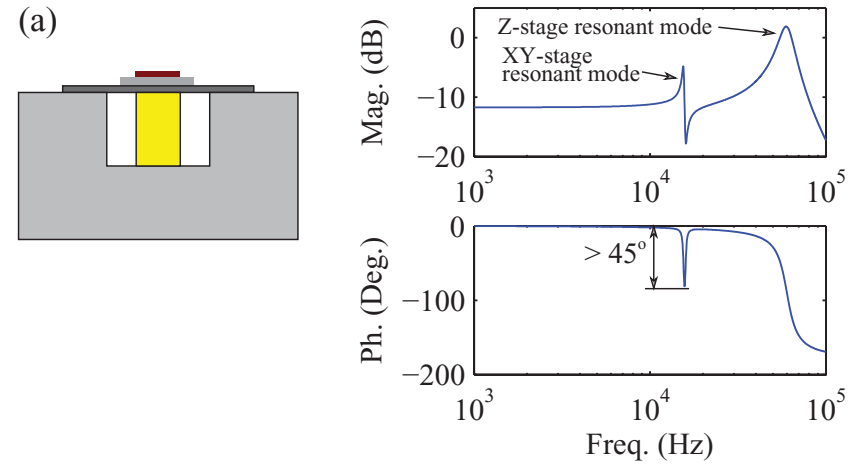

(b1)
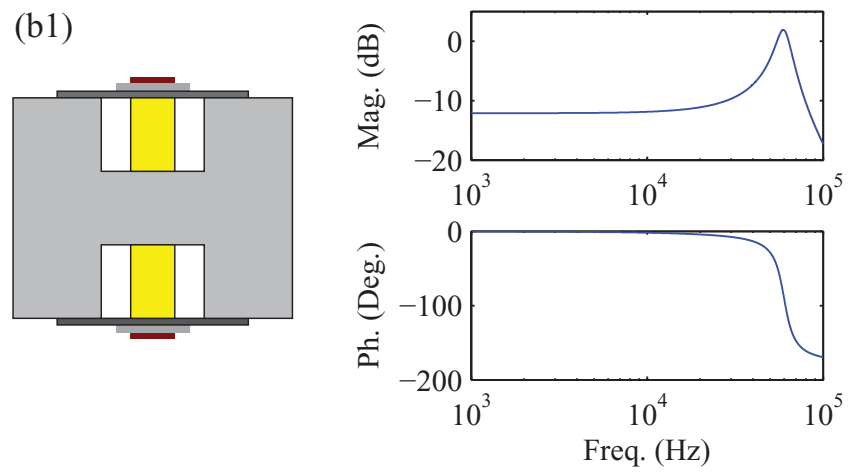

(b2)
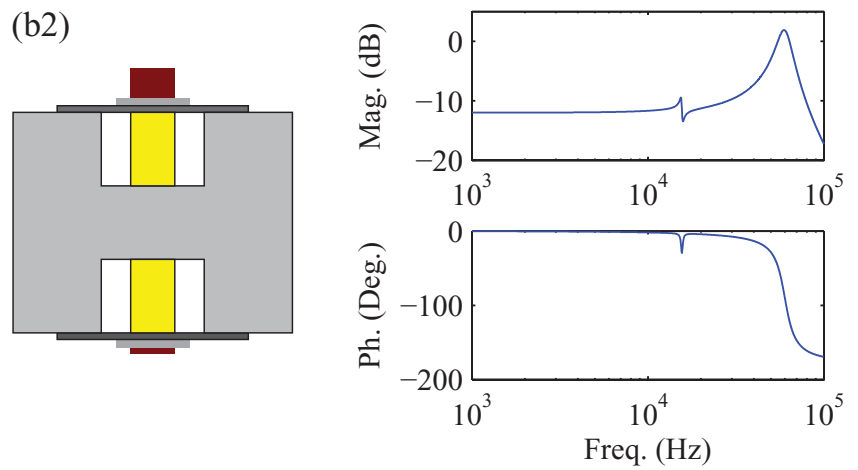

(b3)
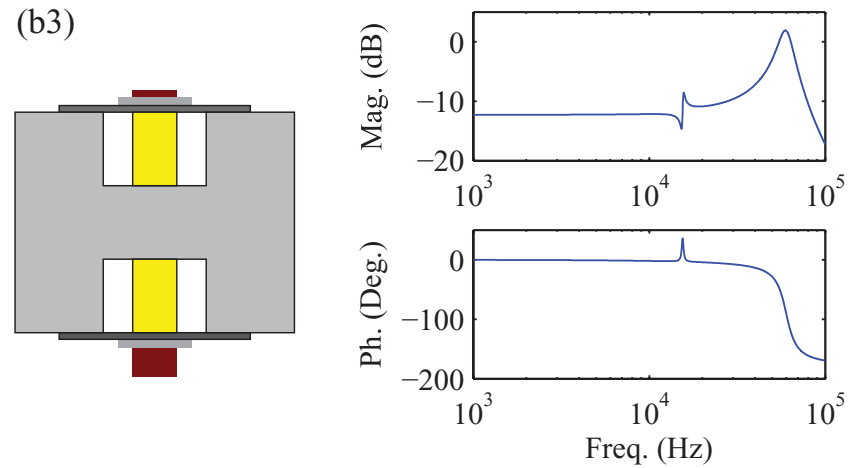

Fig. 9. Frequency responses of different $Z$-nanopositioner configurations. (a) Single-nanopositioner configuration without inertial cancellation. (b) Dualmounted $Z$-nanopositioner configuration: (b1) weights are perfectly balanced; (b2) weight of the sample is slightly heavier than that of the dummy; and (b3) weight of the dummy sample is slightly heavier than that of the sample.

references [22], [39], [40]. When the $Z$-nanopositioner is only comprised of a single nanopositioner, as is the case in [22] and [39] [and illustrated in Fig. 9(a)], the fast movements of 
the piezoelectric stack actuator tend to induce impulsive forces which propagate through, and excite the resonant modes of the $X Y$-nanopositioner [13], [20]. This resonant mode, which often lives at a lower frequency than that of the vertical axis, in turn, causes vibrations in the $Z$-nanopositioner. The frequency response of the single-nanopositioner configuration shows that the vertical resonant mode of the $X Y$-nanopositioner is the dominant mode with a phase drop of more than $45^{\circ}$. This mode may need to be suppressed by means of feedback control to avoid vibrations. In this configuration, the bandwidth of the vertical feedback loop is limited by the low resonance frequency of the $X Y$-stage.

Equation (17) shows that the vertical resonant mode of the $X Y$-stage, $f_{1}$, can be increased by increasing the vertical effective stiffness $k_{s}$ of the $X Y$-nanopositioner. This can be achieved by adding more beam flexures to the $X Y$-nanopositioner. However, these flexures will increase the moving mass of the device which in turn will reduce the resonance frequency. Furthermore, the additional flexures result in a reduction in the $X Y$ - travel range. To avoid this, a dual-mounted $Z$-nanopositioner configuration, illustrated in Fig. 9(b), is used to counterbalance the impulsive forces of the $Z$-actuator. This inertial counterbalance method avoids the need to change $k_{s}$ through a mechanical design. The bottom $Z$-actuator cancels the impulsive force exerted by the top actuator which, when the mass is balanced properly, eliminates the first vertical resonant mode.

The effectiveness of the dual-mounted nanopositioner is highly sensitive to the weight difference between the opposing piezoelectric stack actuators. This is illustrated in Fig. 9. If the sample and the dummy weights are perfectly balanced, the vertical mode of the $X Y$-nanopositioner will not be triggered. In this case, the dominant mode is the vertical mode of the $Z$-nanopositioner where, by design, is at least four times higher than that of the $X Y$-nanopositioner. Thus, in this case, the bandwidth of the vertical feedback loop is limited by the resonant mode of the $Z$-nanopositioner. However, in practice, it is rather difficult to perfectly balance the weights. It is more likely that the dummy sample is slightly lighter or heavier than that of the sample sitting on top of the $Z$-nanopositioner [see Fig. 9(b)]. Nevertheless, if the weights are well balanced, the effect of the $X Y$-stage's dynamics on the vertical positioning stage will be negligible, possibly consisting of a very small peak in magnitude and a phase variation of less than $45^{\circ}$. In such a case, the dynamic mode of the $X Y$-stage will not be the dominant mode.

\section{EXPERIMENTS}

The experimental setup is shown in Fig. 8. A Polytec PSV300 laser scanning vibrometer was used to measure the $Z$-axis frequency response and travel range of the nanopositioner. A 100-Hz, 200-V peak-to-peak sinusoidal signal, which was amplified by a PiezoDrive PDL200 amplifier, was applied to drive the piezoelectric stack actuator, and the measured full-range displacement of the $Z$-nanopositioner is $5 \mu \mathrm{m}$. The displacement is plotted in Fig. 10.

A pseudorandom input signal with a frequency in the range of $1-100 \mathrm{kHz}$ was applied to the piezoelectric stack actuator.
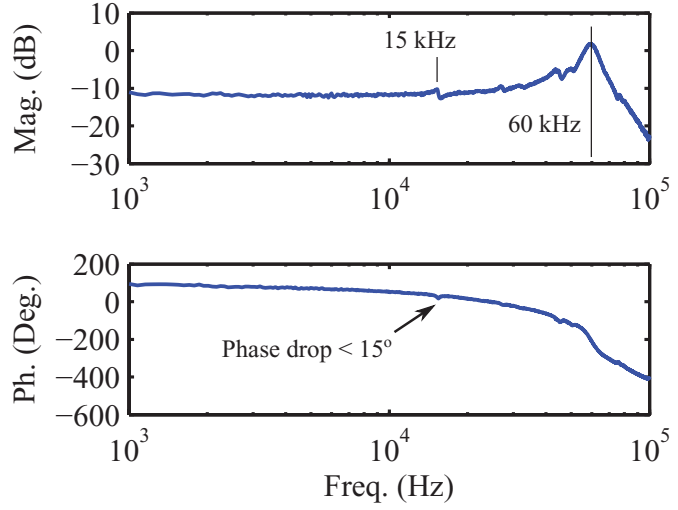

(a)

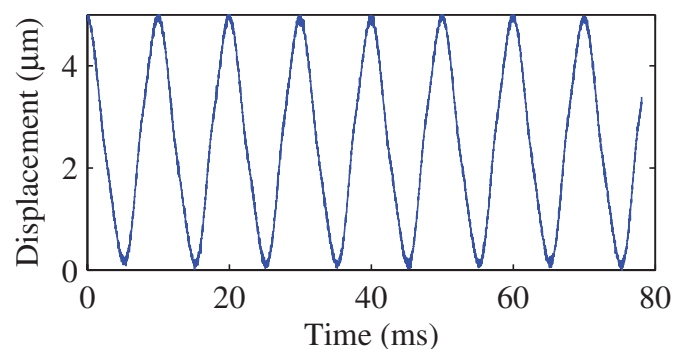

(b)

Fig. 10. (a) Measured frequency response. (b) Travel range of the nanopositioner.

The measured frequency response of the $Z$-nanopositioner is plotted in Fig. 10(a). The weight of the dummy sample is $0.03 \mathrm{~g}$, which is approximately the weight of the sample. The frequency response of the $Z$-nanopositioner in Fig. 10(a) shows a polezero pattern, indicating that the dummy mass is slightly lighter than the sample. The measured first vertical resonant mode of the single-mounted $Z$-nanopositioner is $17.1 \mathrm{kHz}$. Due to the additional weights of the dual-mounted $Z$-nanopositioner, the vertical resonant mode of the $X Y$-nanopositioner drops from 17.1 to $15 \mathrm{kHz}$. However, its resonant peak has a magnitude of only $1.6 \mathrm{~dB}$ and a phase drop of less than $15^{\circ}$. Due to its negligible profile, this mode will not be the dominant mode that dictates the vertical feedback bandwidth. As shown in the measured frequency response, the dominant mode in the $Z$-axis is the $60-\mathrm{kHz}$ resonant peak. As a result, a controller may be designed to suppress this resonant peak in order to obtain an even higher bandwidth.

\section{IMAGE PERFORMANCES}

A commercial NT-MDT NTEGRA AFM was used in our experiments. This AFM uses a piezoelectric tube scanner for nanopositioning, which was replaced by the $X Y Z$ high-speed nanopositioner presented in this study. A $6 \mu \mathrm{m} \times 6 \mu \mathrm{m}$ area of a MikroMasch TGZ2 test grating was scanned during the experiments. This grating weights $0.03 \mathrm{~g}$. It has repeating rectangular features of $3-\mu \mathrm{m}$ period and 108-nm step height. A CSG30 cantilever which has a spring constant of $0.6 \mathrm{~N} / \mathrm{m}$ and a resonance frequency of $76 \mathrm{kHz}$ was used to perform scans. Images were captured in the constant-force contact mode with the vertical 

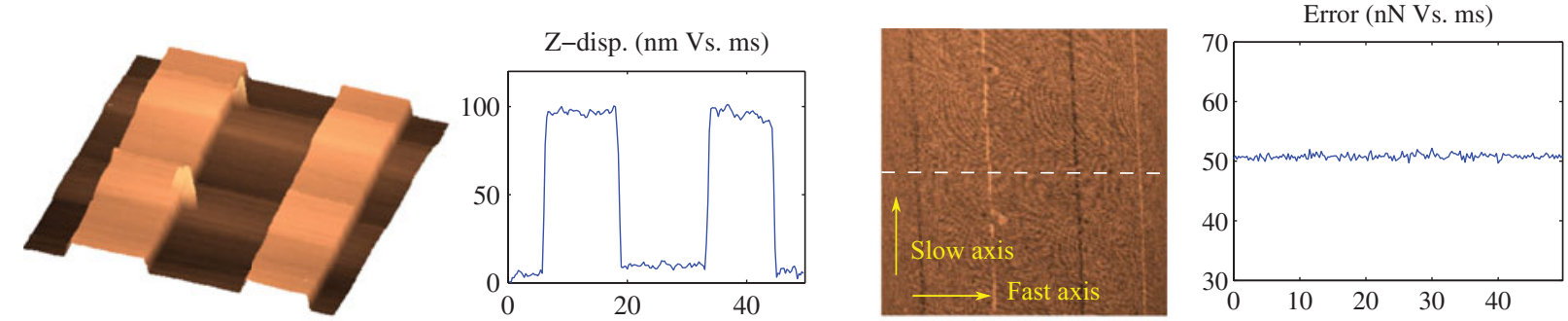

$10 \mathrm{~Hz}(200 \times 200)$
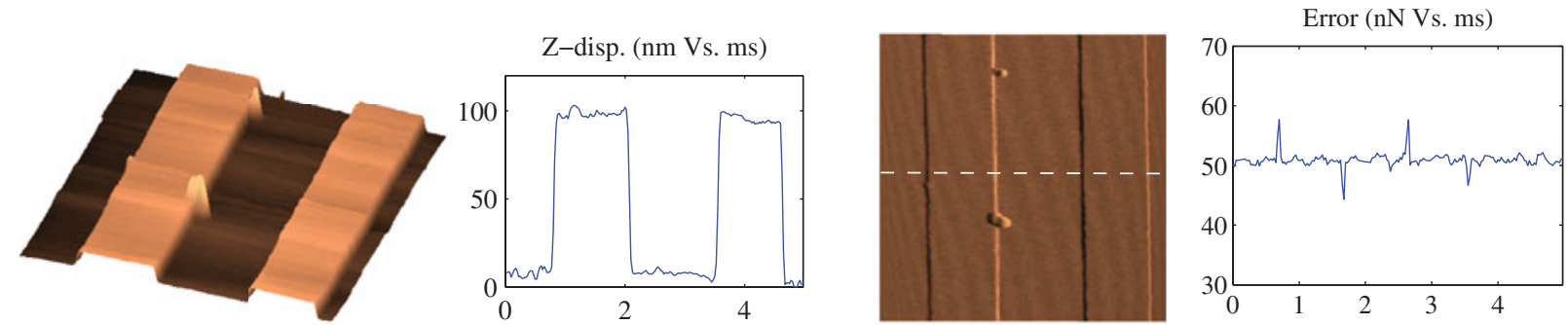

$100 \mathrm{~Hz}(200 \times 200)$
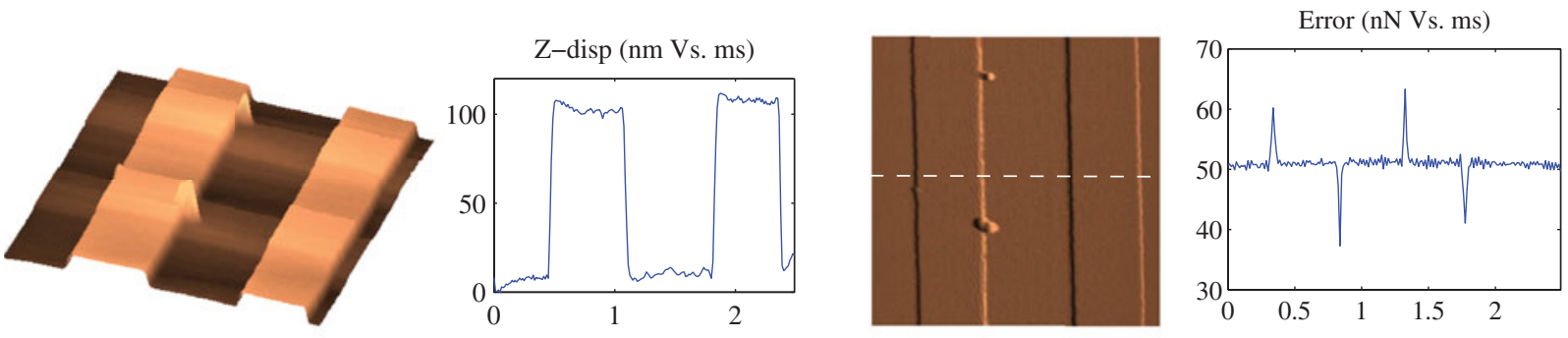

$200 \mathrm{~Hz}(200 \times 200)$
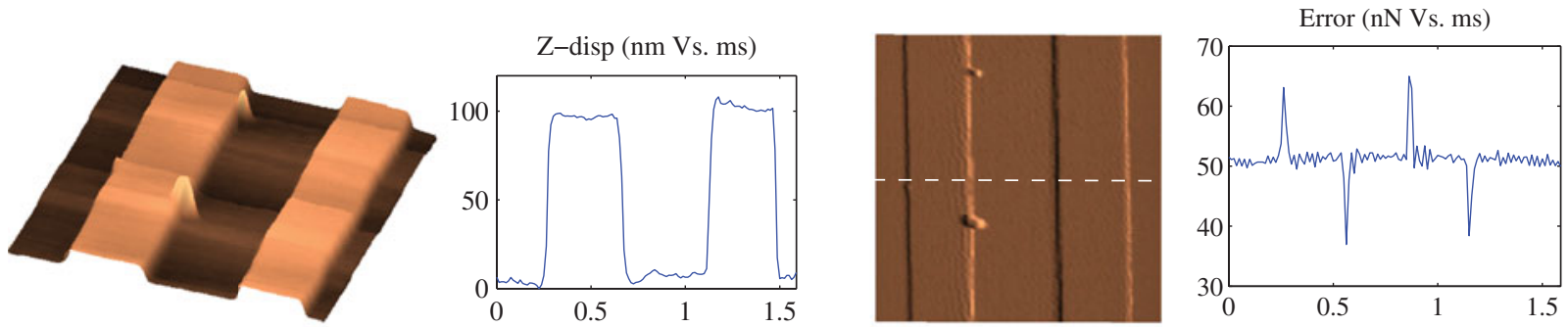

$312 \mathrm{~Hz}(128 \times 128)$
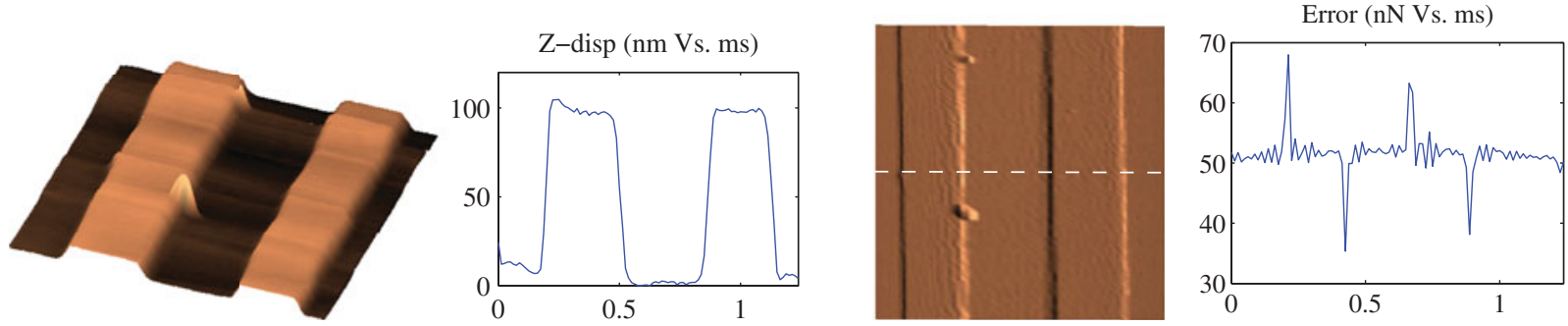

$400 \mathrm{~Hz}(100 \times 100)$

Fig. 11. Scan images ( $6 \mu \mathrm{m} \times 6 \mu \mathrm{m}$ area) acquired by using the proposed $Z$-nanopositioner. From the left: 3-D topographic images of the test grating; single-line $Z$-displacement profiles plotted in $\mathrm{nm}$ versus $\mathrm{ms}$; images plotted using the force error signals; single-line tracking errors in plotted $\mathrm{nN}$ versus $\mathrm{ms}$. 
TABLE II

RMS TRACKING ERRORS OF THE $Z$-AXIS IN NN AT VARIOUS SCAN FREQUENCIES

\begin{tabular}{cc}
\hline \hline Scan freq. (Hz) & RMS error (nN) \\
\hline 10 & 2 \\
100 & 5.9 \\
200 & 8.3 \\
312 & 13.0 \\
400 & 15.4 \\
\hline
\end{tabular}

integral tracking controller activated. The tip-sample interaction force was set to $50 \mathrm{nN}$. The measured closed-loop vertical bandwidth is $6.5 \mathrm{kHz}$ (at $-45^{\circ}$ phase) with gain and phase margins of $4 \mathrm{~dB}$ and $73^{\circ}$, respectively. The AFM's vertical feedback loop was replaced with an external loop to enable a higher integral gain, as is necessary in high-speed scans.

During the scans, the $X$ - and $Y$-axes were operated in open loop. Sinusoidal signals were used to drive the fast axis of the nanopositioner in order to avoid excitation of the lateral resonant modes. Sinusoidal signals have been used in tuning fork AFM [21], [41] and some other high-speed nonraster scan patterns AFM [42], [43]. $200 \times 200$ pixel resolution images were obtained at line rates of 10, 100, and $200 \mathrm{~Hz}$. Fig. 11 shows the scanned images of the test grating, single-line $Z$-displacement plots, images constructed from tracking errors, and the singleline tracking error plots. Images recorded at $100-$ and $200-\mathrm{Hz}$ line rates are of similar quality compared with that at $10 \mathrm{~Hz}$, indicating that the $Z$-nanopositioner has sufficient vertical feedback bandwidth to track sharp edges of the sample at these high scan speeds. Table II documents the RMS tracking errors at these scan speeds. Their RMS errors are within $8.5 \mathrm{nN}$. A similar image quality cannot be achieved using the commercial AFM when operated with its own piezoelectric tube scanner which has a dominant resonance frequency of $680 \mathrm{~Hz}$ and a vertical control bandwidth of only $120 \mathrm{~Hz}$.

The performance of the proposed $Z$-nanopositioner was further tested where images were recorded at $312 \mathrm{~Hz}(128 \times$ 128 pixel resolution) and $400 \mathrm{~Hz}(100 \times 100$ pixel resolution $)$. Note that the image resolution is reduced at high speeds due to the limitation of the data acquisition board. Vibration-induced image artifacts were not observed at these high scan speeds. However, the sharp features of the sample are slightly rounded due to the insufficient vertical feedback bandwidth. This effect can be observed in both 3-D images and $Z$-displacement plots in Fig. 11. RMS errors at these scan speeds are increased to $13 \mathrm{nN}$ and $15.4 \mathrm{nN}$, respectively, indicating the gradual reduction of the tracking performance. Nevertheless, the proposed $Z$-nanopositioner is capable of obtaining images at approximately 200 times faster than that of the commercial piezoelectric tube scanner.

\section{CONCLUSION}

We presented a $Z$-nanopositioner for high-speed atomic force microscopy. The $Z$-nanopositioner utilizes diaphragm flexures to preload the two $Z$-axis piezoelectric stack actuators and to guide the $Z$-axis motions. Stiffness and resonance frequencies of the single-mounted $Z$-nanopositioner were calculated ana- lytically to obtain an in-depth understanding of the static and dynamic behavior of the design. This was followed by using the dual-mounted configuration to counterbalance the impulsive force induced by the $Z$-axis actuators. We demonstrated that the dual-mounted $Z$-nanopositioner effectively avoided the excitation of resonant modes of the $X Y$-nanopositioner. The dual-mounted $Z$-nanopositioner has a displacement range of $5 \mu \mathrm{m}$ and a dominant resonance frequency at $60 \mathrm{kHz}$. This amounts to a closed-loop vertical bandwidth of $6.5 \mathrm{kHz}$. With the high-vertical feedback bandwidth, features with sharp edges were successfully recorded at scan rates up to $200 \mathrm{~Hz}$ without noticeable image artifacts. Future work involves suppression of the vertical axis resonances of the $Z$-nanopositioner by means of feedback control to enable even faster scans.

\section{REFERENCES}

[1] G. Binnig, H. Rohrer, C. Gerber, and E. Weibel, "Surface studies by scanning tunneling microscopy," Phys. Rev. Lett., vol. 49, no. 1, pp. 5761,1982 .

[2] G. Binnig, C. F. Quate, and C. Gerber, "Atomic force microscope," Phys. Rev. Lett., vol. 56, pp. 930-933, 1986.

[3] S. Sundararajan and B. Bhushan, "Development of AFM-based techniques to measure mechanical properties of nanoscale structures," Sens. Actuat. A, Phys., vol. 101, pp. 338-351, 2002.

[4] B. Bhushan, Springer Handbook of Nanotechnology, 2nd ed. New York: Springer-Verlag, 2004.

[5] K.-B. Choi and J. J. Lee, "Passive compliant wafer stage for single-step nano-imprint lithography," Rev. Sci. Instr., vol. 76, pp. 075106-1-0751066, 2005

[6] D. L. White and O. R. Wood, "Novel alignment system for imprint lithography," Rev. Sci. Instr., vol. 18, no. 6, pp. 3552-3556, 2000.

[7] B. A. Wacaser, M. J. Maughan, I. A. Mowat, T. L. Niederhauser, M. R. Linford, and R. C. Davis, "Chemomechanical surface patterning and functionalization of silicon surfaces using an atomic force microscope," Appl. Phys. Lett., vol. 82, no. 5, pp. 808-810, 2003.

[8] S. Gonda, T. Kurosawa, and Y. Tanimura, "Mechanical performances of a symmetrical, monolithic three-dimensional fine-motion stage for nanometrology," Meas. Sci. Technol., vol. 10, pp. 986-993, 1999.

[9] T. Ando, N. Kodera, T. Uchihashi, A. Miyagi, R. Nakakita, H. Yamashita, and K. Matada, "High-speed atomic force microscope for capturing dynamic behavior of protein molecules at work," E-J. Surface Sci. Nanotechnol., vol. 3, pp. 384-392, 2005.

[10] Q. Zou, K. K. Leang, E. Sadoun, M. J. Reed, and S. Devasia, "Control issues in high-speed AFM for biological applications: Collagen imaging example," Asian J. Control, vol. 6, no. 2, pp. 164-178, 2004.

[11] P. Vettiger, G. Cross, M. Despont, U. Drechsler, U. Dürig, B. Gotsmann, W. Häberle, M. A. Lantz, H. E. Rothuizen, R. Stutz, and G. K. Binnig, "The 'milipede'-nanotechnology entering data storage," IEEE Trans. Nanotechnol., vol. 1, no. 1, pp. 39-55, Mar. 2002.

[12] A. Sebastian, A. Pantazi, S. O. R. Moheimani, H. Pozidis, and E. Eleftheriou, "Achieving sub-nanometer precision in a MEMS storage device during self-servo write process," IEEE Trans. Nanotechnol., vol. 7, no. 5, pp. 586-595, Sep. 2008.

[13] T. Ando, T. Uchihashi, and T. Fukuma, "High-speed atomic force microscopy for nano-visualization of dynamic biomolecular processes," Progr. Surface Sci., vol. 83, no. 7-9, pp. 337-437, 2008.

[14] D. Abramovitch, S. Andersson, L. Pao, and G. Schitter, "A tutorial on the mechanisms, dynamics, and control of atomic force microscopes," in Proc. Amer. Contr. Conf., Jul. 2007, pp. 3488-3502.

[15] T. Ando, T. Uchihashi, N. Kodera, D. Yamamoto, A. Miyagi, M. Taniguchi, and H. Yamashita, "High-speed AFM and nano-visualization of biomolecular processes," Pfügers Arch. Eur. J. Physiol., vol. 456, no. 1, pp. 211$225,2008$.

[16] A. Fleming, "Dual-stage vertical feedback for high-speed scanning probe microscopy," IEEE Trans. Contr. Syst. Technol., vol. 19, no. 1, pp. 156165, Jan. 2011.

[17] A. J. Fleming, B. J. Kenton, and K. K. Leang, "Bridging the gap between conventional and video-speed scanning probe microscopes," Ultramicroscopy, vol. 110, no. 9, pp. 1205-1214, 2010. 
[18] S. Kuiper, A. J. Fleming, and G. Schitter, "Dual actuation for high-speed atomic force microscopy," persented at the IFAC Symp. Mechatron. Syst., Boston, MA, Sep. 2010, pp. 220-226.

[19] N. Kodera, H. Yamashita, and T. Ando, "Active damping of the scanner for high-speed atomic force microscopy," Rev. Sci. Instr., vol. 76, no. 5, pp. 053708-1-053708-5, 2005.

[20] B. J. Kenton and K. K. Leang, "Design and control of a three-axis serialkinematic high-bandwidth nanopositioner," IEEE/ASME Trans. Mechatron., vol. 17, no. 2, pp. 356-369, Apr. 2012.

[21] L. M. Picco, L. Bozec, A. Ulcinas, D. J. Engledew, M. Antognozzi, M. A. Horton, and M. J. Miles, "Breaking the speed limit with atomic force microscopy," Nanotechnology, vol. 18, pp. 044030-1-044030-4, 2007.

[22] Y. K. Yong, B. Bhikkaji, and S. O. R. Moheimani, "Analog control of a high-speed atomic force microscope scanner," persented at the IEEE/ASME Int. Conf. Adv. Intell. Mechatron., Budapest, Hungary, Jul. 3-7, 2011, pp. 646-651.

[23] B. J. Kenton, A. J. Fleming, and K. K. Leang, "Compact ultra-fast vertical nanopositioner for improving scanning probe microscope scan speed," Rev. Sci. Instr., vol. 82, no. 12, pp. 123703-1-123703-8, 2011.

[24] J. Kwon, J. Hong, Y.-S. Kim, D.-Y. Lee, K. Lee, S.-M. Lee, and S.-I. Park, "Atomic force microscope with improved scan accuracy, scan speed, and optical vision," Review, vol. 74, no. 10, pp. 4378-4383, 2003.

[25] Y. K. Yong, T.-F. Lu, and D. C. Handley, "Review of circular flexure hinge design equations and derivation of empirical formulations," Precision Eng., vol. 32, no. 2, pp. 63-70, 2008.

[26] Y. Li and Q. Xu, "Modeling and performance evaluation of a flexurebased xy parallel micromanipulator," Mech. Mach. Theor., vol. 44, no. 12, pp. 2127-2152, 2009.

[27] H.C. Liaw and B. Shirinzadeh, "Neural network motion tracking control of piezo-actuated flexure-based mechanisms for micro/nanomanipulation," IEEE/ASME Trans. Mechatron., vol. 14, no. 5, pp. 517-527, Oct. 2009.

[28] Y. K. Yong and T.-F. Lu, "Kinetostatic modeling of 3-RRR compliant micro-motion stages with flexure hinges," Mech. Mach. Theor., vol. 44, no. 6, pp. 1156-1175, 2009.

[29] N. Lobontiu and E. Garcia, "Analytical model of displacement amplification and stiffness optimization for a class of flexure-based compliant mechanisms," Comput. Struct., vol. 81, pp. 2797-2810, 2003.

[30] S. Awtar, "Synthesis and analysis of parallel kinematic $X Y$ flexure mechanisms," Ph.D. dissertation, Massachusetts Institute of Technology, Cambridge, MA, 2003.
[31] V. Shilpiekandula and K. Youcef-Toumi, Dynamic Modeling and Performance Trade-offs in Flexure-based Positioning and Alignment Systems. New York: InTech, 2010, no. 25, pp. 481-496.

[32] D. Vukobratovich, R. M. Richard, J. P. McNiven, and R. L. Sinclair, "Slit diaphragm flexures for optomechanics," in Proc. SPIE: Optomech. Precision Instr. Des., 1995, vol. 2542, pp. 2-10.

[33] F. Beer and E. R. Johnston, Mechanics of Materials. New York: McGraw-Hill, 1992.

[34] N. Lobontiu, Compliant Mechanisms: Design of Flexure Hinges. Boca Raton, FL: CRC Press, 2003.

[35] T. Dahlberg, "Procedure to calculate deflections of curved beams," Int. J. Eng. Edu., vol. 20, no. 3, pp. 503-513, 2004.

[36] G. Schitter, K. J. Åstrom, B. DeMartini, P. J. Thurner, K. L. Turner, and P. K. Hansma, "Design and modeling of a high-speed AFM-scanner," IEEE Trans. Contr. Syst. Technol., vol. 15, no. 5, pp. 906-915, Sep. 2007.

[37] The World of Micro- and NanoPositioning (PI catalog). Physik Instrumente, Karlsruhe, Germany, 2005

[38] Y. K. Yong, S. Aphale, and S. O. R. Moheimani, "Design, identification, and control of a flexure-based $X Y$ stage for fast nanoscale positioning," IEEE Trans. Nanotechnol., vol. 8, no. 1, pp. 46-54, Jan. 2009.

[39] Y. K. Yong and S. O. R. Moheimani, "A compact XYZ scanner for fast atomic force microscopy in constant force contact mode," persented at the IEEE/ASME Int. Conf. Adv. Intell. Mechatron., Montreal, Canada, Jul. 6-9, 2010, pp. 225-230.

[40] Y. K. Yong and S. O. R. Moheimani, "Design, modeling and FPAAbased control of a high-speed atomic force microscope nanopositioner," IEEE/ASME Trans. Mechatron., to be published. DOI: 10.1109/TMECH.2012.2194161

[41] A. D. L. Humphris, J. K. Hobbs, and M. J. Miles, "Ultrahigh-speed scanning near-field optical microscopy capable of over 100 frames per second," Appl. Phys. Lett., vol. 83, no. 1, pp. 6-8, 2003.

[42] I. A. Mahmood, S. O. R. Moheimani, and B. Bhikkaji, "A new scanning method for fast atomic force microscopy," IEEE Trans. Nanotechnol., vol. 10, no. 2, pp. 203-216, Mar. 2010.

[43] Y. K. Yong, S. O. R. Moheimani, and I. R. Petersen, "High-speed cycloidscan atomic force microscopy," Nanotechnology, vol. 21, no. 36, pp. 365503-1-365503-4, 2010.

Authors' photographs and biographies not available at the time of publication. 